\title{
Nursery school children's observation responses to novel, complex, and asymmetrical complex stimuli
}

THOMAS J. BANTA, JUNE SCIARRA ANO JEAN JETT

UNIV'ERSITY OF CINCINNATI

Children's observation responses to novel stimuli was found to be a function of (1) adaptation level induced by repeated presentations of a simple stimulus, (2) complexity of the stimulus, (3) asymmetry of the stimulus for some Ss, and (4) individual differences on a "stimulus differentiation" dimension. Retesting a month later showed these functions to be stable.

Research and theory support the generalization that complex and novel stimuli are attended to longer than simple stimuli and that a recurring stimulus is not attended to as long as a changing stimulus (Berlyne, 1958; Fantz, 1964; Welker, 1961; Mendel, 1965). The present experiment was designed to test these generalizations with three to six year old children and to examine two other questions: (1) Is differential sensitivity to complex stimuli stable over a one month test-retest interval by three years of age? (2) Does the three to five year old child differentiate asymmetrical from symmetrical complex stimuli?

\section{Subjects}

The Ss were 33 Negro children ranging in age between two years seven months and six years of age, average age being four years two months. The 18 female and 15 male children were enrolled in a community nursery school in Cincinnati, Ohio.

\section{Experimental design}

The experimental procedure was designed to stabilize the Ss' complexity adaptation level (Helson, 1959) by repeatedly presenting a simple standard stimulus (a red block) on repeated trials throughout the experiment. Three kinds of critical stimuli were interposed in the stimulus series: (1) "simple novel" stimuli blocks of different shape or color than the standard, not differing in complexity from the standard stimulus, (2) "complex" stimuli - symmetrical, but complex three-dimensional objects, and (3) "complex asymmetrical" stimuli - three-dimensional objects not symmetrically organized.

The stability of average stimulus effects and the stability of individual differences was tested by administering the stimulus series approximately one month after the first testing. The test-retest interval averaged 27.6 days, with $82 \%$ of the children tested within a 24 to 29 day interval.

\section{Description of stimuli and stimulus sequence}

The standard stimulus was a red wooden block, 3-1/2 $\mathrm{x}$ $1-3 / 4 \times 7 / 8$ in. This was presented three times prior to each critical stimulus. The simple novel stimuli appeared on trials 12,24 , and 36 ; the complex stimuli appeared on trials 4,16 , and 28; and the asymmetrical complex stimuli appeared on trials 8,20 , and 32 . The critical stimuli are illustrated in Fig. 1 and are described below in their order of appearance:

Stimulus 4, complex - a triangular white Styrofoam block, sides 6-1/2 $\times 4 \times 4$ in. with eight pink plastic pins inserted upright and parallel equidistantly around the edge to a height of $2-3 / 4$ in.;

Stimulus 8 , asymmetrical complex -16 in. of coat hanger wire bent at irregular intervals with five wooden beads (two red, two yellow, one green), one placed at each bend of the wire;

Stimulus 12, simple novel - small red wooden block $1-3 / 4 \times 1-3 / 4 \times 7 / 8$ in.;

Stimulus 16 , complex -6 in. paper plate painted with yellow circle in center, a red circle around the yellow, and a black circle around the red, all perfectly concentric;

Stimulus 20, asymmetrical complex - an abstract clay figure, reddish-pink in color, with several protrusions and closed loops extending from the base, approximately $2-1 / 4 \mathrm{in}$. high and $2 \mathrm{in}$. in diameter;

Stimulus 24 , simple novel - yellow wooden block $1-3 / 4 \times 1-3 / 4 \times 7 / 8$ in.;

Stimulus 28, complex - pale green plastic coated paper tray $8-1 / 2 \times 6-1 / 4$ in. with $1 / 2$ in. edges with five $10-1 / 2$ in. pastel straws extending parallel and equidistantly across bottom and through sides of tray;

Stimulus 32 , complex asymmetrical -6 in. diameter $45 \mathrm{rpm}$ phonograph record heated to irregular shape with half a plastic egg glued off center (except for the asymmetry, this object is matched with Stimulus 16, with the half egg being yellow, the record label painted red, and the black record surface on the irregularly melted edge);

Stimulus 36 , simple novel - yellow wooden block $1-3 / 4$ $\times 3 / 4 \times 3 / 4$ in.

\section{Procedure}

A $14 \times 9-1 / 2$ in. box, 5-1/2 in. high was used for presentation of the stimulus series. Cellophane covered the top of the box to prevent the child's handling the objects. The bottom of the box was removed so that $\mathrm{E}$ 


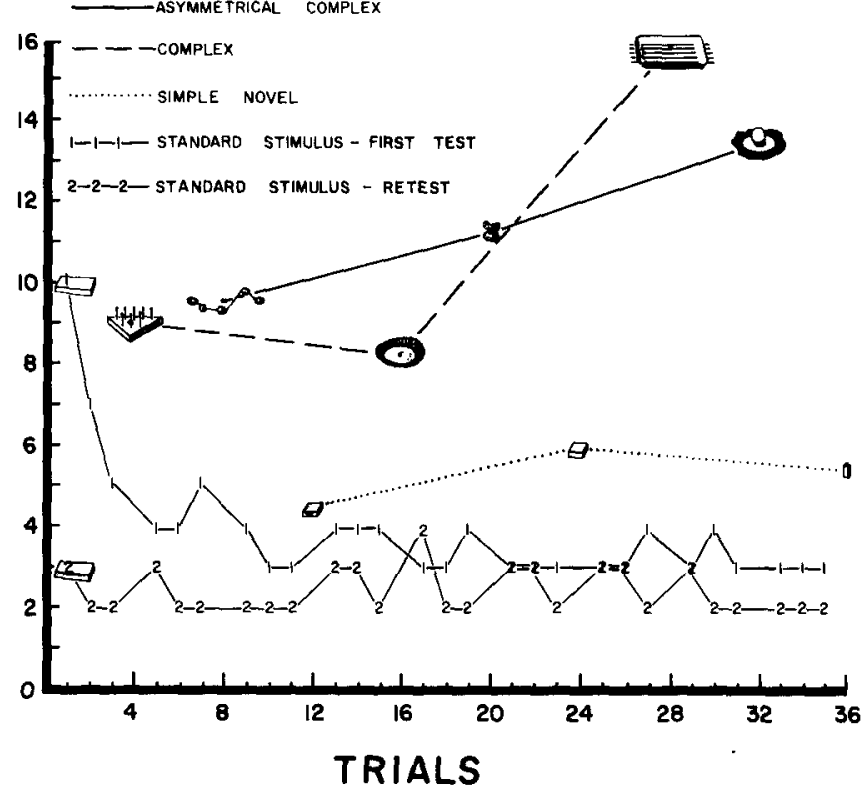

Fig. 1. Duration of observation responses for test and retest data. Response durations for critical stimuli were not significantly different for test and retest measurements; these data are combined in the figure. Only the standard stimuli ( 1 and 2 ) are presented separately for test and retest conditions. could put the stimuli in the box. A lid closed over the cellophane cover and was hinged at the rear $9-1 / 2$ in. edge of the box. S sat opposite $\mathrm{E}$. The instructions were: "This is a special kind of box. It has a lid that you can lift up to look in." E demonstrates lifting the lid. "You can look at something in the box but you cannot touch it because there is paper on the top. Would you like to lift the lid and look in the box?" (E pauses while $S$ looks at box and is satisfied and familiar with how the lid works.) "I am going to put something in the box for you to look at. You may look at it as long as you want to. When you are finished, put the lid down. Now close your eyes while I put something in the box." $\mathrm{E}$ puts the red block in the box and says, "Now you may look." E starts the stop watch when the child looks into the box. When $\mathrm{S}$ looks away or looks at $\mathrm{E}$ the watch is stopped and started again only when $S$ is looking into the box again. $E$ looks at child with same facial expression after each lid closing, waits 2 sec., says "Close your eyes," and then records time and puts next object in the box. Only rarely did the child lift the lid again for another look, at which time E said, "When you put the lid down you must leave it down."

\section{Results}

Stimulus effects. Durations of observation responses to the standard, simple, novel, complex, and asymmetrical complex stimuli are given in Fig. 1. Test and retest data for the standard stimulus series are presented separately. Standard stimulus response durations were longer at the outset of the first testing and dropped sharply. By trial three the durations approached asymptote and only gradual decrease was observed. The test and retest curves were very similar beyond trial 12 . The retest curve for the standard stimulus shows that there were short observation response durations at the outset that continued throughout the experimental series.

Critical stimulus effects present during the first testing were present in the same degree during the second testing. Since no test-retest differences were significant for the critical stimuli, these data have been averaged for presentation in Fig. 1. These averaged data for all Ss showed no significant differences between complex and asymmetrical complex stimuli. However, responses to both classes of complex stimuli were longer than responses to the simple novel stimuli. Eighty-five percent of the Ss on the first testing and $82 \%$ on retest looked longer at the complex stimuli than at the simple novel stimuli.

Test-retest stability of individual differences. In addition to the stability of average stımulus effects, it was possible to evaluate the stability across individual Ss in terms of differential responsiveness to critical stimuli. A ratio was computed based on total response duration for critical stimuli and total responseduration for the standard stimulus. Thus a child who was a high differentiator looked much longer at critical stimuli than he looked at the standard stimulus. The highest differentiator in the sample looked 8.46 times longer at the critical stimuli than he did at the standard stimulus. The lowest differentiator actually looked somewhat less at critical stimuli than at the standard stimulus presentations.

The overall test-retest correlation for this differentiation score was +.18 and was not statistically significant. Examination of the scatter plot revealed that the younger children accounted for the low correlation. Eliminating the youngest third of the sample (three years and ten months or younger) resulted in a correlation of $+.41(p<.05)$. 
Individual differences and complex us. asymmetrical complex stimuli. On the assumption that Ss perceived the differences between the three classes of critical stimuli used in the experiment, it was expected that duration of observation responses would be ordered from shortest to longest responses in the following way in relation to the three classes of experimental stimuli: (1) simple novel, (2) complex, and (3) asymmetrical complex. It turned out, however, that only $45 \%$ of all Ss responded in this way. Thirty-six percent responded in one other unexpected order, (1) simple novel, (2) asymmetrical complex, and (3) complex. The remaining $18 \%$ were accounted for by four other unexpected orders.

Using the data from the testing a month earlier, Ss were classified as high, medium, and low differentiators. High differentiators, those Ss who looked longer at all critical stimuli than at the standard stimulus, showed highly predictable response patterns: $73 \%$ gave the expected ordering of response durations, differentiating between simple novel, complex, and asymmetrical stimuli. Medium differentiators showed less predictability: only $36 \%$ gave the expected ordering. Low differentiators were even less predictable: only $27 \%$ gave expected orderings.

\section{Discussion}

Observation response durations were shown to vary as a function of novelty and complexity for nursery school children. Observation responses to a simple standard stimulus, repeated throughout the series, adapted rapidly and remained brief throughout the first experimental series and showed no sign of recovery of its novelty vaiue when presented in the second experimental series a month later. Critical stimuli, interposed in the standard stimulus series, were observed for nearly identical durations on both the first and retest experimental series. Thus, it is apparent that the critical stimuli remained relatively novel while the repeated stimulus lost, and did not regain its novelty. Complexity adaptation level (determined in the present study by the recurrent presentations of a simple standard stimulus) stabilized early in the first experiment and was maintained throughout the retest a month later.

All children in the present study had seen, handled, and played with blocks in nursery school, but the first presentation of a common red block in a new experimental setting commanded as long an abservation response as a much more novel and complex stimulus presented later in the series. After adapting, however, the simple novel stimuli comprised of other sizes or colors were observed longer than the standard stimulus, but not as long as more complexly organized stimuli.

An analysis of the physical properties of these stimuli shows that while they all vary in many dimensions (color, contour, number of components, etc.) the data are more parsimoniously regarded as due to variations in the (1) simplicity-complexity dimension and (2) degree of shost-term novelty relative to the standard stimulus.

Differential responding to complex vs. asymmetrical complex stimuli in the present experiment was present for those Ss who were high differentiators. Medium and low differentiators did not show the organization of perception in terms of symmetry-asymmetry properties of the stimuli. This individual difference factor in perception introduced here very likely reflects both genetic-structural predisposition as well as later stimulus enrichment experiences.

Evaluation of enrichment nursery school programs might well include this perceptual organization assessment procedure. It is reasonable to expect that enrichment intervention programs should have an effect on perceptual responsiveness to novel and complex stimuli. The children with greater curiosity who are differentially sensitive to stimulus dimensions of simplicity-complexity, or symmetry-asymmetry in relation to background stimulation are children who promise to be more sensitive problem-solvers and more interested in the visual textures and contours of the external world.

\section{References}

Berlyne, D. E. The influence of complexity and rovelty in visuas fígures on orientitg responses. J. exp. Psychol., 1958, 55 289-296.

Fantz, R. L. Visual experience in infants: decreased attention to familiar patterns relative to novel ones. Science, 1964, 146, 668-670.

Helson, H. Adaptation level theory. In S. Koch (Ed.), Psychology: A study of a science. Vol. 1. Sensory, perceptual, and physiological foundations. New York: McGraw-Hill, 1959. Pp. 565-621.

Mendel, Gisela. Children's preferences for differing degrees of novelty. Child Develpm., 1965, 36, 453-465.

Welker, W. 1. An analysis of exploratory and play behavior in animals. In D. W. Fiske, \& S. R. Maddi (Eds.), Functions Df varied experience. Homewood, Illinois: Dorsey Press, 1961. Pp $175-226$

\section{Note}

1. The authors want to thank Miss Louise Cuyler, Director of the Wesley Child Care Center, and her Head Teacher, Mis Dorothy Stroud, who so effectively helped coordinate teachers, children, and researchers in this study.

(Received in the Editorial Office April 4, 1966.) 